\title{
Pareto Type-II Model under Type-I Progressive Hybrid Censoring: Bound Lengths
}

\author{
Gyan Prakash \\ Department of Community Medicine, \\ M. L. N. Medical College, Allahabad, U. P., India.
}

\begin{abstract}
The Pareto Type-II model is considered here from which, the observable is to be predicted by using Bayesian approach. The Bayes prediction bound lengths are obtained for Type-I progressive hybrid censored data. Both One-sample and Two-sample Bayes prediction scenario has included in the present study. Both known and unknown cases of the scale parameter have considered in the present study. A comparison also has made with the asymptotic interval estimates, are made-up from the Fisher information matrix. Performance of the different methods has studied by simulation and a real data set.
\end{abstract}

Keywords: type-I progressive hybrid censoring, approximate confidence length (ACL), onesample (OS-BPBL), two-sample (TS-BPBL) Bayes prediction bound lengths.

\section{Introduction}

The underlying distribution is a mixture of the Exponential distribution with scale parameter $\alpha$, and the given scale parameter $\alpha$ is distributed as Gamma density with parameters $\theta$ and $\sigma$. The probability density function of the considered Pareto distribution is

$$
f(x ; \sigma, \theta)=\theta \sigma^{\theta}(x+\sigma)^{-\theta-1} ; x \geq 0, \theta>0, \sigma>0 .
$$

The considered Pareto distribution is also called as Lomax distribution or the Pareto Type-II distribution. In given Eq. (1), the parameter $\theta$ is called as shape parameter, whereas the parameter $\sigma$ is known as the scale parameter.

In a flexible family of fat-tailed distributions, the Pareto distribution take a good place and is a valuable model for the income distribution of the higher and complex income group. The Lomax model also plays a vital role in socioeconomic studies and is frequently used as a model for examining areas including city population distribution, stock price variation, oil field sites and armed force areas etc. The Pareto distribution has frequently used distribution for model survival after some medical procedures due to its decreasing failure rate.

Lomax (1954), Steindle (1965), Harries (1967), Madi and Raqab (2004), and others have discussed about the applicability and usefulness of the Pareto distribution in a variety of other problems, like as size of cities \& firms, business mortality, service time in the queuing 
system and forecasting of the temperature records etc. Several authors have discussed time to time about properties of the Pareto distribution in different aspects. Few recent literatures on the underlying distribution is stated here.

Okasha (2014) concerned in his article about E-Bayesian method for computing the estimates of the reliability parameters of the Lomax distribution by using conventional Type-II censored data. Based on the right ordered sample data, central coverage bound lengths under the Bayesian inferences was inspected by Prakash (2014) for the Lomax model. Under the Jeffrey's and Gamma prior, Ahmad, Ahmad, and Ahmed (2016) discussed some classical and Bayes estimation by using Entropy and Precautionary loss functions.

Recently, Prakash (2017) was discussed about some statistical inference based on Progressive Type-II censored data for two-parameter Pareto distribution. Using a compound LINEX asymmetric loss function, the Bayes estimation and E-Bayes estimation of Lomax parameter has studied recently by Wei, Wang, and Li (2017).

The goal of the present study is to investigate the properties of Bayes prediction bound lengths for unknown parameters of the underlying distribution. Two different scenarios have used for predicting future observations on Type-I Progressive hybrid censored data for known $\&$ unknown cases of scale parameter. The performances of the procedures are illustrated by a simulation technique based on Metropolis-Hastings (M-H) algorithm and a real data example was discussed by Chhikara and Folks (1977). A comparison also has been made with the asymptotic interval estimates obtained from Fisher information matrix by following Park and Balakrishnan (2009).

\section{Type-I progressive hybrid censoring}

Numerous authors have investigated several lifetime models using the hybrid censoring scheme. A little few of them are stated here. Kundu and Joarder (2006) obtained the maximum likelihood and Bayes estimators for unknown parameters of the Exponential distribution along with different confidence intervals. Some useful classical estimators for the unknown shape and scale parameters of the Weibull distribution under Type-I progressive hybrid censoring scheme have discussed by Lin, Ng, and Chan (2009). Singh, Gupta, and Sharma (2014) presents some estimation for the Lindley distribution using Type-II hybrid censored data. Recently, Kayal, Tripathi, Rastogi, and Asgharzadeh (2017) discussed some inferences for the Burr Type-XII distribution under Type-I progressive hybrid censoring. See Balakrishnan and Cramer (2014) for more details about the hybrid censoring scheme. In the present article, some inferences based on Bayesian approach have discussed for the Pareto Type-II distribution by using Type-I progressive hybrid censored data.

In this article, the prediction of future failures on Type-I progressive hybrid censored data for Pareto Type-II distribution has studied. One-Sample and Two-Sample Bayes prediction technique have applied for the prediction of future failures. The Bayesian approaches proposed in this article is also different from the existing ones. Here, we used the Metropolis-Hastings algorithm to compute the corresponding credible intervals numerically by the help of Monte Carlo simulations technique. A comparison with the asymptotic interval estimates have discussed also.

In conventional Type-I and Type-II censoring, a life test is terminated at a prescribed time span or at a predefined number of failures. The main drawback of these censoring schemes is, the units cannot be removed from the test at any time point except the final closure point. However, the progressive Type-II censoring scheme gives the flexibility of eliminating of the test units before the final termination.

On other hand, the major drawback of the progressive Type-II censoring is that, it can take a lot of time to reach the final termination point (Kundu and Joarder (2006)). Kundu and Joarder (2006) have discussed about Type-I progressive hybrid censoring scheme which is the result of the mixture of progressive Type-II and conventional Type-I (time censoring) schemes. In Type-I progressive hybrid censoring scheme, the life tests stops when a predefined number 
of failures occurred or when a prescribed time on the test has reached.

Let us suppose, total of $n$ identical test units are subject to a life test experiment with $R \equiv\left(R_{1}, R_{2}, \ldots, R_{m}\right)$ be the prescribed censoring scheme follows the assumption $n-m=$ $R_{1}+R_{2}+\ldots+R_{m}$. The predefined time point $t$ is also fixed. When, the first failure $X_{1: n: m}$ (say) occurs, the $R_{1}$ of the remaining units are randomly removed from the experiment. Similarly, for the second failure $X_{2: n: m}, R_{2}$ of the remaining units are randomly removed and so on. If the $m^{t h}$ failure occurs before a prescribed time point $t$, then the experiment is terminated at the time point $X_{m: n: m}$.

In case, the experiment stops at time $t$ with number of failures $X_{j: n: m}$, then they must satisfying the condition $X_{j: n: m}<t<X_{j+1: n: m}$ and remaining all the live test units $n-R_{1}-$ $R_{2}-\ldots-R_{j}-j=R *$ (say) are removed from the test. Hence, the life test is terminated at random time $t_{1}=\min \left(X_{m: n: m}, t\right)$. So, the observed sample may be one of the following two types:

$$
\begin{cases}\text { Case } I(I): \text { say }:\left(X_{1: n: m}, X_{2: n: m}, \ldots, X_{m: n: m}\right) & \text { if } X_{m: n: m}<t \\ \text { Case II }:\left(X_{1: n: m}, X_{2: n: m}, \ldots, X_{j: n: m}\right) & \text { if } X_{j: n: m}<t<X_{j+1: n: m} .\end{cases}
$$

Under the Type-I progressive hybrid censoring scheme, the joint probability density function is defined as

$$
\left\{\begin{array}{l}
\text { Case I : } L(\underline{x} \mid \sigma, \theta) \propto \prod_{i=1}^{m} f\left(x_{(i)} ; \sigma, \theta\right)\left(1-F\left(x_{(i)} ; \sigma, \theta\right)\right)^{R_{i}} ; x_{(i)} \equiv X_{i: n: m} \\
\text { Case II }: L(\underline{x} \mid \sigma, \theta) \propto \prod_{i=1}^{j} f\left(x_{(i)} ; \sigma, \theta\right)\left(1-F\left(x_{(i)} ; \sigma, \theta\right)\right)^{R_{i}}(1-F(t ; \sigma, \theta))^{R *}
\end{array}\right.
$$

Using Eq. (1) in Eq. (2), we get

$$
L(\underline{x} \mid \sigma, \theta)=\theta^{d} e^{-T_{0}(\underline{x}, \sigma)} e^{-\theta T(\underline{x}, \sigma)}
$$

where

$$
\left\{\begin{array}{l}
\text { Case I : } T(\underline{x}, \sigma)=\sum_{i=1}^{m}\left(1+R_{i}\right) \log \left(x_{i}+\sigma\right)-n \log \sigma \\
\text { Case II }: T(\underline{x}, \sigma)=\sum_{i=1}^{j}\left(1+R_{i}\right) \log \left(x_{i}+\sigma\right)-(n+R *) \log \sigma+R * \log (t+\sigma),
\end{array}\right.
$$

$T_{0}(\underline{x}, \sigma)=\sum_{i=1}^{d} \log \left(x_{i}+\sigma\right)$ and and $d(=m)$ for Case I \& $d(=j)$ for Case II.

\section{Bayes prediction bound length (known scale parameter)}

In many industrial applications, one is often challenged to give estimates for upcoming observation on the basis of any prior knowledge. Another main side of such study are the calculation of prediction lengths of the unknown observable. In this section, we obtained Bayes predictive bound lengths based on One-Sample and Two-Sample technique under the Type-I progressive hybrid censoring.

In this section, we assume scale parameter $\sigma$ is known first. The natural family of prior distribution is considered under the above assumption as the two parameter Gamma distribution, having probability density

$$
\pi(\theta \mid \sigma)=\frac{\sigma^{\alpha}}{\Gamma(\alpha)} \theta^{\alpha-1} e^{-\theta \sigma} ; \alpha>0, \sigma>0, \theta>0 .
$$

The posterior distribution corresponding to the parameter $\theta$ is thus obtained as

$$
\pi_{\theta}^{*}=\frac{(\sigma+T(\underline{x}, \sigma))^{\alpha+d}}{\Gamma(\alpha+d)} \theta^{\alpha+d-1} e^{-\theta(\sigma+T(\underline{x}, \sigma))} .
$$




\subsection{One-sample Bayes prediction technique}

Let $X \equiv\left(x_{1}, x_{2}, \ldots, x_{d}\right)$ be the Type-I progressive hybrid censored sample with the censoring scheme $R \equiv\left(R_{1}, R_{2}, \ldots, R_{d}\right)$ from the considered model Eq. (1). If $Y \equiv\left(y_{1}, y_{2}, \ldots, y_{d}\right)$ be the second independent random sample of future observations from the same model. Then the Bayes predictive density for the future observation $Y$, is denoted by $h(y \mid \underline{x})$ and obtained as

$$
\begin{aligned}
& h(y \mid \underline{x})=\int_{\theta} f(y ; \sigma, \theta) \cdot \pi_{\theta}^{*} d \theta \\
&=\frac{(\sigma+T(\underline{x}, \sigma))^{\alpha+d}}{\Gamma(\alpha+d)(y+\sigma)} \int_{\theta} \theta^{\alpha+d} e^{-\theta(\sigma+T(\underline{x}, \sigma)+\log (y+\sigma)-\log \sigma)} d \theta \\
& \Rightarrow h(y \mid \underline{x})=\frac{(\alpha+d)(\sigma+T(\underline{x}, \sigma))^{\alpha+d}}{(y+\sigma)(\sigma+T(\underline{x}, \sigma)+\log (y+\sigma)-\log \sigma)^{\alpha+d+1}} .
\end{aligned}
$$

Let us suppose $l_{1}$ and $l_{2}$ be the lower and upper Bayes prediction bound limits, then $100(1-\epsilon) \%$ Bayes prediction bound length for the future observation $Y$ corresponding to the parameter $\theta$, under Type-I progressive hybrid censoring scenario is obtained by solving following equation

$$
L_{\text {One }}=\left[P\left(Y \geq l_{2}\right)=\frac{\epsilon}{2}\right]-\left[P\left(Y \leq l_{1}\right)=\frac{\epsilon}{2}\right] .
$$

Using Eq. (6) in Eq. (7), the BPBL under one-sample technique for Type-I progressive hybrid censoring is obtained as

$$
L_{\text {One }}=\sigma\left\{e^{(\sigma+T(\underline{x}, \sigma))\left(\epsilon_{2}-1\right)}-e^{(\sigma+T(\underline{x}, \sigma))\left(\epsilon_{1}-1\right)}\right\}
$$

where $\epsilon_{1}=\left(1-\frac{\epsilon}{2}\right)^{-1 /(\alpha+d)}$ and $\epsilon_{2}=\left(\frac{\epsilon}{2}\right)^{-1 /(\alpha+d)}$.

\subsection{Two-sample Bayes prediction technique}

Since, from previous subsection, it was assumed that $X \equiv\left(x_{1}, x_{2}, \ldots, x_{d}\right)$ be the first (observed) Type-I progressive hybrid censored samples with the censoring scheme $R \equiv\left(R_{1}, R_{2}, \ldots, R_{d}\right)$ from the considered model Eq. (1). If $Y \equiv\left(y_{1}, y_{2}, \ldots, y_{d}\right)$ be the second (unobserved) Type-I progressive hybrid censored samples, drawn independently from the same model. Then in two-sample technique the first sample is referred as the instructive or past sample, while the second is mentioned as the future sample. Based on an instructive sample, the $j^{\text {th }}$ order statistic from the future sample will be predicted. The cumulative predictive density $G(y \mid \underline{x})$ under Two-Sample Bayes prediction technique is obtained by using Bayes predictive density given in Eq. (6), of the future observation $Y$ as

$$
G(y \mid \underline{x})=\operatorname{Pr}(Y \leq y)=1-\left\{1+\frac{\log (y+\sigma)-\log \sigma}{\sigma+T(\underline{x}, \sigma)}\right\}^{-\alpha-d} .
$$

If we assume $Y_{j}$ be the $j^{\text {th }}$ order statistic from the future sample of size $m(1 \leq j \leq m)$. Then the probability density function of $j^{t h}$ ordered future observation is defined as

$$
\phi\left(y_{j}\right)=j\left({ }^{m} C_{j}\right)(G(y \mid \underline{x}))^{j-1}(1-G(y \mid \underline{x}))^{m-j} \cdot h(y \mid \underline{x}) .
$$

Using Eq. (7), the Bayes prediction bound length for the $j^{\text {th }}$ future observation under the Two-Sample scenario is obtained by solving following equality as

$$
L_{T w o}=\left[P\left(Y \geq l_{2 j}\right)=\frac{\epsilon}{2}\right]-\left[P\left(Y \leq l_{1 j}\right)=\frac{\epsilon}{2}\right] .
$$


Here, again $l_{2 j} \& l_{1 j}$ be the upper and lower Bayes prediction limits in Two-Sample approach for the $j^{\text {th }}$ future observation. The Bayes prediction bound length for the smallest future observation $j(=1)$ and the last future observation $j(=m)$ are obtained from Eq. (11) by using Eq. $(9-10)$ and given as

$$
L_{T w o(j=1)}=\sigma\left\{\exp \left(\left(\epsilon_{2}^{1 / m}-1\right)(\sigma+T(\underline{x}, \sigma))\right)-\exp \left(\left(\epsilon_{1}^{1 / m}-1\right)(\sigma+T(\underline{x}, \sigma))\right)\right\}
$$

and

$$
L_{T w o(j=m)}=\sigma\left\{e^{\lambda_{2}(\sigma+T(\underline{x}, \sigma))}-e^{\lambda_{1}(\sigma+T(\underline{x}, \sigma))}\right\} ;
$$

where $\lambda_{1}=\left\{\left[1-\left(\frac{\epsilon}{2}\right)^{1 / m}\right]^{-1 /(\alpha+d)}-1\right\}$ and $\lambda_{2}=\left\{\left[1-\left(1-\frac{\epsilon}{2}\right)^{1 / m}\right]^{-1 /(\alpha+d)}-1\right\}$.

\section{Bayes prediction bound length (unknown scale parameter)}

Prakash (2017) assumed the joint prior density function for the Pareto Type-II distribution when both parameters are assumed as the random variable, and is given as

$$
\pi_{(\theta, \sigma)} \propto \theta^{\alpha-1} e^{-\sigma \theta} \sigma^{\alpha+\beta-1} e^{-\gamma \sigma} ; \alpha>0, \beta>0, \gamma>0, \sigma>0, \theta>0 .
$$

The joint posterior and marginal posterior densities corresponding to the parameters $\theta$ and $\sigma$ are obtained under the Type-I progressive hybrid censoring scheme as

$$
\begin{gathered}
\pi_{(\theta, \sigma)}^{*}=\Omega \theta^{\alpha+d-1} e^{-T_{0}(\underline{x}, \sigma)} e^{-\theta(\sigma+T(\underline{x}, \sigma))} \sigma^{\alpha+\beta-1} e^{-\gamma \sigma} \\
\pi_{\theta}^{* *}=\Omega \theta^{\alpha+d-1} \int_{\sigma} \sigma^{\alpha+\beta-1} e^{-\gamma \sigma} e^{-T_{0}(\underline{x}, \sigma)} e^{-\theta(\sigma+T(\underline{x}, \sigma))} d \sigma
\end{gathered}
$$

and

$$
\pi_{\sigma}^{* *}=\Omega \frac{\Gamma(\alpha+d)}{(\sigma+T(\underline{x}, \sigma))^{\alpha+d}} \sigma^{\alpha+\beta-1} e^{-\gamma \sigma} e^{-T_{0}(\underline{x}, \sigma)}
$$

where $\Omega=\left\{\Gamma(\alpha+d) \int_{\sigma} \frac{e^{-\gamma \sigma} e^{-T_{0}(\underline{x}, \sigma)}}{(\sigma+T(\underline{x}, \sigma))^{\alpha+d}} \sigma^{\alpha+\beta-1} d \sigma\right\}^{-1}$.

\subsection{One-sample Bayes prediction technique}

On similar lines, the Bayes predictive density function $\tilde{h}(y \mid \underline{x})$ for the future observation $Y$ under Type-I progressive hybrid censored sample for the parameters $\Theta(=\theta, \sigma)$ are obtained as

$$
\tilde{h}_{\Theta}(y \mid \underline{x})=\int_{\Theta} \theta \frac{e^{\theta \log \sigma}}{y+\sigma} e^{-\theta \log (y+\sigma)} \cdot \pi_{\Theta}^{* *} d \Theta .
$$

If $l_{1}$ and $l_{2}$ be the lower and upper Bayes prediction bound limits, then 100(1- $\left.\epsilon\right) \%$ Bayes prediction bound limits for the future observation $Y$ under Type-I progressive hybrid censoring corresponding to the parameters $\Theta(=\theta, \sigma)$, are obtained by solving following inequality

$$
\begin{gathered}
\left(1-\frac{\epsilon}{2}\right)=\int_{\Theta} e^{-\theta \log \left(\frac{l_{1}+\sigma}{\sigma}\right)} \cdot \pi_{\Theta}^{* *} d \Theta, \\
\left(\frac{\epsilon}{2}\right)=\int_{\Theta} e^{-\theta \log \left(\frac{l_{2}+\sigma}{\sigma}\right)} \cdot \pi_{\Theta}^{* *} d \Theta,
\end{gathered}
$$

and

$$
L_{\text {One }}=l_{2}-l_{1} .
$$


The values corresponding to $l_{1} \& l_{2}$ can be obtained by solving Eq. (19) \& Eq. (20) respectively with corresponding parameters $\Theta(=\theta, \sigma)$.

\subsection{Two-sample Bayes prediction technique}

On similar lines as discussed in the previous section, the cumulative predictive density $\tilde{G}_{\Theta}(y \mid \underline{x})$ under Two-Sample Bayes prediction scheme on Type-I progressive hybrid censoring scheme is obtained for the future observation $Y$ from Eq. (18) as

$$
\tilde{G}_{\Theta}(y \mid \underline{x})=\operatorname{Pr}(Y \leq y)=\int_{\Theta}\left\{1-e^{-\theta \log \left(\frac{y+\sigma}{\sigma}\right)}\right\} \cdot \pi_{\Theta}^{* *} d \Theta .
$$

The probability density function of the $j^{\text {th }}$ ordered future observation is given as

$$
\tilde{\phi}\left(y_{j}\right)=j\left({ }^{m} C_{j}\right)\left(\tilde{G}_{\Theta}(y \mid \underline{x})\right)^{j-1}\left(1-\tilde{G}_{\Theta}(y \mid \underline{x})\right)^{m-j} \tilde{h}_{\Theta}(y \mid \underline{x}) .
$$

One may obtain the solution of Eq. (23) by substituting Eq. (18) \& Eq. (22) respectively, for both parameters. However, the closed form of the Eq. (23) for either parameter does not exist. If, $l_{2 j} \& l_{1 j}$ be the upper and lower Bayes prediction limits in Two-Sample approach for the $j^{\text {th }}$ future observation, then the Bayes prediction bound length under Two-Sample approach can be obtained by solving Eq. (11). The simplified forms of the Bayes prediction bound length for the smallest future observation $j(=1)$ and the largest future observation $j(=m)$ are not possible to obtain from Eq. (11) by using Eq. (23). Numerical technique is applied herewith for the numerical illustration.

\section{Asymptotic interval estimates}

The joint probability function based on Type-I progressive hybrid censoring pattern is defined in Eq. (3). The logarithm of Eq. (3) is given as

$$
\log L(\underline{x} \mid \sigma, \theta)=d \log \theta-T_{0}(\underline{x}, \sigma)-\theta T(\underline{x}, \sigma) .
$$

Differentiating Eq. (24) with respect to the parameters separately and equating to zero, we get

$$
\frac{\partial}{\partial \theta} \log L(\underline{x} \mid \sigma, \theta)=\frac{d}{\theta}-T(\underline{x}, \sigma)
$$

and

(For Case I:)

$$
\frac{\partial}{\partial \sigma} \log L(\underline{x} \mid \sigma, \theta)=\sum_{i=1}^{m}\left(\frac{1}{x_{(i)}+\sigma}\right)-\theta\left\{\sum_{i=1}^{m}\left(\frac{1+R_{i}}{x_{(i)}+\sigma}\right)-\frac{n}{\sigma}\right\}
$$

(For Case II:)

$$
\frac{\partial}{\partial \sigma} \log L(\underline{x} \mid \sigma, \theta)=\sum_{i=1}^{j}\left(\frac{1}{x_{(i)}+\sigma}\right)-\theta\left\{\sum_{i=1}^{j}\left(\frac{1+R_{i}}{x_{(i)}+\sigma}\right)-\frac{n+R *}{\sigma}+\frac{R *}{t+\sigma}\right\} .
$$

The maximum likelihood estimators corresponding to the parameters $\theta$ and $\sigma$ are denoted respectively by $\hat{\theta}_{M l}$ and $\hat{\sigma}_{M l}$, and obtained as

$$
\hat{\theta}_{M l}=\frac{d}{T(\underline{x}, \sigma)}
$$

and

$$
\hat{\sigma}_{M l}=h(\sigma)(s a y)
$$


where

Case I:

$$
h(\sigma)=\frac{n \theta}{\theta \sum_{i=1}^{m}\left(\frac{1+R_{i}}{x_{(i)}+\sigma}\right)-\sum_{i=1}^{m}\left(\frac{1}{x_{(i)}+\sigma}\right)}
$$

Case II:

$$
h(\sigma)=\frac{\theta(n+R *)}{\theta\left(\frac{R *}{t+\sigma}\right)+\theta \sum_{i=1}^{j}\left(\frac{1+R_{i}}{x_{(i)}+\sigma}\right)-\sum_{i=1}^{j}\left(\frac{1}{x_{(i)}+\sigma}\right)} .
$$

A simple iterative procedure have adopted for solving Eq. (26). For this let $\sigma^{(0)}$ is an initial guess value of $\sigma$. Then successive approximations of $\sigma$ are $\sigma^{(1)}=h\left(\sigma^{(0)}\right), \sigma^{(2)}=h\left(\sigma^{(1)}\right), \ldots$, $\sigma^{(m+1)}=h\left(\sigma^{(m)}\right)$. Stop the iterative procedure at the $m^{t h}$ stage if $\left|\sigma^{(m+1)}-\sigma^{(m)}\right|<\varphi$, for some pre-specified tolerance limit $\varphi$. Once, the ML estimate $\hat{\sigma}_{M l}$ obtained then the ML estimate $\hat{\theta}_{M l}$ easily obtained from Eq. (25).

The Fisher Information matrix is redefined by Park and Balakrishnan (2009), for the hybrid censoring scheme, and is given as

$$
l(\Theta)=\left[\begin{array}{ll}
l_{11}(\Theta) & l_{12}(\Theta) \\
l_{21}(\Theta) & l_{22}(\Theta)
\end{array}\right] .
$$

where

$$
\begin{gathered}
l_{11}(\Theta)=\int_{0}^{t}\left\{\frac{\partial}{\partial \theta} \log \rho\right\}^{2} \sum_{i=1}^{m} f_{i: n}(x ; \Theta) d x \\
l_{22}(\Theta)=\int_{0}^{t}\left\{\frac{\partial}{\partial \sigma} \log \rho\right\}^{2} \sum_{i=1}^{m} f_{i: n}(x ; \Theta) d x \\
l_{12}(\Theta)=l_{21}(\Theta)=\int_{0}^{t}\left\{\frac{\partial}{\partial \theta} \log \rho\right\}\left\{\frac{\partial}{\partial \sigma} \log \rho\right\} \sum_{i=1}^{m} f_{i: n}(x ; \Theta) d x .
\end{gathered}
$$

Here, $\rho$ and $f_{i: n}(x ; \Theta)$ are known as the hazard function for Eq. (1) and the probability density of $X_{i: n}$ respectively, and are defined as

$$
\rho=\frac{\theta}{x+\sigma}
$$

and

$$
\begin{gathered}
f_{i: n}(x ; \Theta)=i\left({ }^{n} C_{i}\right)(F(x ; \sigma, \theta))^{i-1}(1-F(x ; \sigma, \theta))^{n-i} f(x ; \sigma, \theta) \forall i=1,2, \ldots, m \\
\Rightarrow f_{i: n}(x ; \Theta)=i\left({ }^{n} C_{i}\right)\left(1-\left(\frac{\sigma}{x+\sigma}\right)^{\theta}\right)^{i-1} \frac{\theta \sigma^{\theta(n-i+1)}}{(x+\sigma)^{\theta(n-i+1)+1}} .
\end{gathered}
$$

Hence,

$$
\begin{aligned}
& l_{11}(\Theta)=\sum_{i=1}^{m} i\left({ }^{n} C_{i}\right) \int_{0}^{t}\left(1-\left(\frac{\sigma}{x+\sigma}\right)^{\theta}\right)^{i-1} \frac{\sigma^{\theta(n-i+1)}}{\theta(x+\sigma)^{\theta(n-i+1)+1}} d x \\
& l_{22}(\Theta)=\sum_{i=1}^{m} i\left({ }^{n} C_{i}\right) \int_{0}^{t}\left(1-\left(\frac{\sigma}{x+\sigma}\right)^{\theta}\right)^{i-1} \frac{\theta \sigma^{\theta(n-i+1)}}{(x+\sigma)^{\theta(n-i+1)+3}} d x
\end{aligned}
$$

and

$$
l_{12}(\Theta)=l_{21}(\Theta)=\sum_{i=1}^{m} i\left({ }^{n} C_{i}\right) \int_{0}^{t}\left(1-\left(\frac{\sigma}{x+\sigma}\right)^{\theta}\right)^{i-1} \frac{\sigma^{\theta(n-i+1)}}{(x+\sigma)^{\theta(n-i+1)+2}} d x
$$


It is clear that the elements of $l(\Theta)$ from Eq. (27) are to be computed numerically.

\section{Metropolis-Hastings (M-H) algorithm and simulation analysis}

Metropolis, Rosenbluth, Rosenbluth, Teller, and Teller (1953) and Hastings (1970) has stated an algorithm named as Metropolis-Hastings algorithm. M-H algorithm offers a substitute way for analyzing Bayesian inference and, is mostly used to simulate samples from a given posterior distribution by making use of an arbitrary proposal distribution.

Let us assume, both parameters $\theta$ and $\sigma$ of the underlying distribution given in Eq. (1), be independently distributed as normal. In order to simulate replicates from prescribed distribution, we must implements following steps

1. Set an initial guess of $\delta=(\theta, \sigma)$, say $\delta_{0}$ and defined as $\delta_{0}=\left(\theta_{0}, \sigma_{0}\right)$.

2. In next step, generates a new $\delta^{\prime}$ by using the proposal $N\left(\delta_{n-1}, \sum\right)$ distribution. Here $n$ is the iterative stage and $\sum$ is the variance-covariance matrix.

3. Now, calculate

$$
h=\min \left\{1, \frac{\pi_{\left(\delta^{\prime}\right)}^{*}}{\pi_{\left(\delta_{n-1}\right)}^{*}}\right\} .
$$

4. Now, from Uniform distribution $U(0,1)$, generate a sample $u$ which satisfies

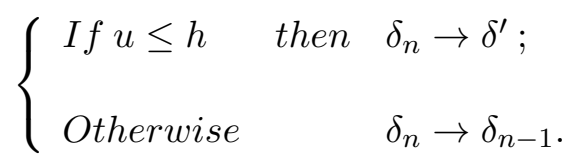

5. Repeat above steps up to $N$ times and collect adequate number of replicates.

In the previous sections, by utilizing One-Sample \& Two-Sample Bayes prediction technique we proposed Bayes Prediction bound lengths under the Type-I progressive hybrid censoring. Both known and unknown cases of scale parameter have considered in the discussion. Assessing the behavior by using Type-I progressive hybrid censored samples. A Monte Carlo simulations on the basis of 10,000 replications have been used under the $\mathrm{M}-\mathrm{H}$ algorithm as discussed above.

\begin{tabular}{|c|c|c|c|c|c|c|c|c|}
\hline \multicolumn{3}{|c|}{$\theta=2.00, m \rightarrow$} & \multicolumn{3}{|c|}{10} & \multicolumn{3}{|c|}{15} \\
\hline$t$ & $\sigma$ & $\alpha \downarrow \epsilon \rightarrow$ & $99 \%$ & $95 \%$ & $90 \%$ & $99 \%$ & $95 \%$ & $90 \%$ \\
\hline \multirow{6}{*}{04} & \multirow{3}{*}{0.25} & 0.50 & 1.0832 & 1.0811 & 1.0422 & 1.0354 & 1.0333 & 0.9962 \\
\hline & & 1.10 & 1.1884 & 1.1778 & 1.1455 & 1.1361 & 1.1258 & 1.0952 \\
\hline & & 1.50 & 1.0821 & 1.0565 & 0.9192 & 1.0341 & 1.0098 & 0.8785 \\
\hline & \multirow{3}{*}{0.50} & 0.50 & 0.9784 & 0.9693 & 0.9414 & 0.9352 & 0.9266 & 0.8998 \\
\hline & & 1.10 & 1.0596 & 1.0468 & 1.0234 & 1.0131 & 1.0007 & 0.9782 \\
\hline & & 1.50 & 0.9716 & 0.9451 & 0.9029 & 0.9287 & 0.9032 & 0.8951 \\
\hline \multirow{6}{*}{07} & \multirow{3}{*}{0.25} & 0.50 & 1.0579 & 1.0558 & 1.0179 & 1.0112 & 1.0093 & 0.9729 \\
\hline & & 1.10 & 1.1608 & 1.1503 & 1.1189 & 1.1096 & 1.0996 & 1.0696 \\
\hline & & 1.50 & 1.0568 & 1.0321 & 0.8979 & 1.0101 & 0.9864 & 0.8583 \\
\hline & \multirow{3}{*}{0.50} & 0.50 & 0.9556 & 0.9468 & 0.9194 & 0.9134 & 0.9049 & 0.8789 \\
\hline & & 1.10 & 1.0351 & 1.0225 & 0.9996 & 0.9894 & 0.9775 & 0.9555 \\
\hline & & 1.50 & 0.9491 & 0.9231 & 0.9164 & 0.9072 & 0.8824 & 0.8654 \\
\hline
\end{tabular}

Table 1: Bound length for parameter $\theta$ under one-sample scenario

The Bayes prediction bound lengths for the parameter $\theta$ under One-Sample technique are presented in Table (1) whereas the bound length under Two-Sample technique has presented 
in Table (2) when scale parameter $\sigma$ is considered to be known. When both the parameters are random variable, the findings are presented in Tables (3) - (5). Prakash (2014) studied the Bayes estimators of the concerned distribution under the right censored data and noted that, the risks are minimized for the smaller scale parameter $\sigma$. Thus, in the present study the pre assumed values of $\sigma$ being smaller and assumed as $0.25 \& 0.50$ whereas the value of $\theta$ is fixed at $\theta(=2.00)$.

Table 2: Bound length for parameter $\theta$ under two-sample scenario

\begin{tabular}{|c|c|c|c|c|c|c|c|c|c|}
\hline \multicolumn{4}{|c|}{$\theta=2.00$} & \multicolumn{3}{|c|}{ First Future Observation } & \multicolumn{3}{|c|}{ Last Future Observation } \\
\hline$m$ & $t$ & $\sigma$ & $\alpha \downarrow \epsilon \rightarrow$ & $99 \%$ & $95 \%$ & $90 \%$ & $99 \%$ & $95 \%$ & $90 \%$ \\
\hline \multirow{12}{*}{10} & \multirow{6}{*}{04} & \multirow{3}{*}{0.25} & 0.50 & 0.9521 & 0.9406 & 0.8783 & 1.3267 & 1.3218 & 1.2664 \\
\hline & & & 1.10 & 1.0491 & 1.0292 & 0.9698 & 1.4591 & 1.4436 & 1.3953 \\
\hline & & & 1.50 & 0.9504 & 0.9179 & 0.7717 & 1.3234 & 1.2894 & 1.1134 \\
\hline & & \multirow{3}{*}{0.50} & 0.50 & 0.8588 & 0.8423 & 0.7923 & 1.1976 & 1.1845 & 1.1432 \\
\hline & & & 1.10 & 0.9345 & 0.9138 & 0.8657 & 1.3003 & 1.2825 & 1.2461 \\
\hline & & & 1.50 & 0.8522 & 0.8198 & 0.7578 & 1.1873 & 1.1523 & 1.0934 \\
\hline & \multirow{6}{*}{07} & \multirow{3}{*}{0.25} & 0.50 & 0.9324 & 0.9212 & 0.8603 & 1.2976 & 1.2929 & 1.2387 \\
\hline & & & 1.10 & 1.0275 & 1.0079 & 0.9498 & 1.4271 & 1.4119 & 1.3649 \\
\hline & & & 1.50 & 0.9309 & 0.8993 & 0.7563 & 1.2944 & 1.2615 & 1.0894 \\
\hline & & \multirow{3}{*}{0.50} & 0.50 & 0.8415 & 0.8253 & 0.7764 & 1.1716 & 1.1589 & 1.1184 \\
\hline & & & 1.10 & 0.9156 & 0.8954 & 0.8481 & 1.2723 & 1.2547 & 1.2191 \\
\hline & & & 1.50 & 0.8352 & 0.8033 & 0.7721 & 1.1617 & 1.1274 & 1.1122 \\
\hline \multirow{12}{*}{15} & \multirow{6}{*}{04} & \multirow{3}{*}{0.25} & 0.50 & 0.9096 & 0.8985 & 0.8392 & 1.2677 & 1.2631 & 1.2101 \\
\hline & & & 1.10 & 1.0026 & 0.9835 & 0.9267 & 1.3946 & 1.3796 & 1.3338 \\
\hline & & & 1.50 & 0.9078 & 0.8767 & 0.7373 & 1.2643 & 1.2319 & 1.0636 \\
\hline & & \multirow{3}{*}{0.50} & 0.50 & 0.8205 & 0.8047 & 0.7569 & 1.1443 & 1.1319 & 1.0924 \\
\hline & & & 1.10 & 0.8931 & 0.8733 & 0.8271 & 1.2431 & 1.2258 & 1.1909 \\
\hline & & & 1.50 & 0.8141 & 0.7829 & 0.7511 & 1.1344 & 1.1008 & 1.0838 \\
\hline & \multirow{6}{*}{07} & \multirow{3}{*}{0.25} & 0.50 & 0.8909 & 0.8804 & 0.8221 & 1.2401 & 1.2358 & 1.1838 \\
\hline & & & 1.10 & 0.9818 & 0.9632 & 0.9078 & 1.3642 & 1.3495 & 1.3047 \\
\hline & & & 1.50 & 0.8893 & 0.8595 & 0.7226 & 1.2369 & 1.2053 & 1.0412 \\
\hline & & \multirow{3}{*}{0.50} & 0.50 & 0.8039 & 0.7884 & 0.7418 & 1.1196 & 1.1074 & 1.0693 \\
\hline & & & 1.10 & 0.8755 & 0.8557 & 0.8105 & 1.2162 & 1.1994 & 1.1653 \\
\hline & & & 1.50 & 0.7979 & 0.7674 & 0.7286 & 1.1101 & 1.0773 & 1.0497 \\
\hline
\end{tabular}

The simulation is carried out for a particular set of Type-I progressive hybrid censored data of size $n(=20)$, with different choices of $m, t$ and level of significance $\epsilon$. The progressive censoring plans for $m(=10)$ and $m(=15)$ are given as 1100110141 and 020110024101310 with pre-assumed values of hyper parameter $\alpha=0.50(0.10) 1.50$.

It is observed from the Table (1) is that, the OS-BPBL first increases when the hyper parameter $\alpha$ increases and maximizes at $\alpha=1.10$ and then decreases. The bound length becomes closer when censored sample size $m$ increases. Similar trend have also seen for pre-assumed values of $\sigma$ or pre-determined failure time $t$. Further, the wider bound length has founds for the wider level of significance. That's shows that as the significance level go to narrower bound lengths also goes into narrower.

Table (2), presents the Two-Sample Bayes prediction bound lengths for the similar preassumed parametric values under the Type-I progressive hybrid censored data for the shape parameter $\theta$ when scale parameter $\sigma$ is known. The bound lengths for the smallest and largest both future observation have presented. The difference between bound lengths for the largest and smallest future observations are not much wider. It is observed also that the largest future observation shows almost wider bound lengths as compared to OS-BPBL. Other properties are seen to be similar. 
Table 3: One-sample Bayes prediction bound length when both parameter unknown

\begin{tabular}{|c|c|c|c|c|c|c|c|c|}
\hline \multicolumn{3}{|c|}{$\theta=2.00, m \rightarrow$} & \multicolumn{3}{|c|}{10} & \multicolumn{3}{|c|}{15} \\
\hline$t$ & $\sigma$ & $\alpha \downarrow \epsilon \rightarrow$ & $99 \%$ & $95 \%$ & $90 \%$ & $99 \%$ & $95 \%$ & $90 \%$ \\
\hline \multicolumn{9}{|c|}{ Bound Length for Shape Parameter $\theta$} \\
\hline \multirow{6}{*}{04} & \multirow{3}{*}{0.25} & 0.50 & 1.1199 & 1.1083 & 1.0673 & 1.0611 & 1.0581 & 1.0213 \\
\hline & & 1.10 & 1.2216 & 1.2101 & 1.0985 & 1.1664 & 1.1569 & 1.1246 \\
\hline & & 1.50 & 1.1174 & 1.0807 & 0.9397 & 1.0585 & 1.0335 & 0.8677 \\
\hline & \multirow{3}{*}{0.50} & 0.50 & 1.0218 & 0.9921 & 0.9638 & 0.9573 & 0.9481 & 0.9209 \\
\hline & & 1.10 & 1.0778 & 1.0744 & 1.0506 & 1.0399 & 1.0259 & 1.0045 \\
\hline & & 1.50 & 0.9905 & 0.9559 & 0.9219 & 0.9473 & 0.9227 & 0.9148 \\
\hline \multirow{6}{*}{07} & \multirow{3}{*}{0.25} & 0.50 & 1.0849 & 1.0815 & 1.0428 & 1.0358 & 1.0336 & 0.9964 \\
\hline & & 1.10 & 1.1925 & 1.1814 & 1.1484 & 1.1405 & 1.1292 & 1.0985 \\
\hline & & 1.50 & 1.0817 & 1.0569 & 0.8185 & 1.0326 & 1.0089 & 0.8773 \\
\hline & \multirow{3}{*}{0.50} & 0.50 & 0.9785 & 0.9691 & 0.9413 & 0.9351 & 0.9259 & 0.8995 \\
\hline & & 1.10 & 1.0629 & 1.0497 & 1.0264 & 1.0158 & 1.0033 & 0.9809 \\
\hline & & 1.50 & 0.9707 & 0.9464 & 0.9372 & 0.9275 & 0.9016 & 0.8846 \\
\hline \multicolumn{9}{|c|}{ Bound Length for Scale Parameter $\sigma$} \\
\hline \multirow{6}{*}{04} & \multirow{3}{*}{0.25} & 0.50 & 1.1659 & 1.1413 & 1.0887 & 1.1045 & 1.0894 & 1.0416 \\
\hline & & 1.10 & 1.2732 & 1.2477 & 1.1217 & 1.2155 & 1.1927 & 1.1484 \\
\hline & & 1.50 & 1.1626 & 1.1121 & 0.9575 & 1.1011 & 1.0633 & 0.8838 \\
\hline & \multirow{3}{*}{0.50} & 0.50 & 1.0635 & 1.0212 & 0.9828 & 0.9961 & 0.9758 & 0.9389 \\
\hline & & 1.10 & 1.1233 & 1.1075 & 1.0727 & 1.0835 & 1.0574 & 1.0255 \\
\hline & & 1.50 & 1.0301 & 0.9831 & 0.9393 & 0.9856 & 0.9488 & 0.9324 \\
\hline \multirow{6}{*}{07} & \multirow{3}{*}{0.25} & 0.50 & 1.1293 & 1.1136 & 1.0636 & 1.0781 & 1.0641 & 1.0161 \\
\hline & & 1.10 & 1.2428 & 1.2186 & 1.1727 & 1.1885 & 1.1641 & 1.1217 \\
\hline & & 1.50 & 1.1253 & 1.0875 & 0.8335 & 1.0745 & 1.0379 & 0.8937 \\
\hline & \multirow{3}{*}{0.50} & 0.50 & 1.0183 & 0.9975 & 0.9597 & 0.9729 & 0.9528 & 0.9174 \\
\hline & & 1.10 & 1.1075 & 1.0819 & 1.0479 & 1.0583 & 1.0343 & 1.0014 \\
\hline & & 1.50 & 1.0094 & 0.9733 & 0.9549 & 0.9643 & 0.9275 & 0.9011 \\
\hline
\end{tabular}

Table (3) - (5), presents the bound lengths for both the parameters under the considered scenario when scale parameter is considered to be random variable. All the properties have seen similar as discussed above. However, one remarkable point is that, the magnitude of the bound lengths becomes wider as compared to the known scale parameter case when other parameters are considered to be fixed.

Table (6), presents the asymptotic confidence length (ACL), for selected parametric values as discussed above. Again, the behavior of all selected parameters have seen similar as discussed above. However, the magnitude of ACL have seen narrower when compared with OS-BPBL. It is also observed that, the ACL for the parameter $\sigma$ are narrower as compared to ACL for the parameter $\theta$.

\section{Study on real data}

A real-life data set is considered here, to illustrate the proposed method and verify how our estimators work in practice. Chhikara and Folks (1977) used 46 observations for the repair times of airborne communication transceiver given in Table (7). In present discussion, we considered only 20 observations from that data set. The validity of the Lomax model was checked again by using Kolmogrov-Smirnov (K-S) test, Anderson-Darling (A-D) and chi-square tests.

We have fitted the Lomax distribution by using $\theta=2.00$ and $\sigma=0.25$, and it is observed that $\mathrm{K}-\mathrm{S}=0.0825$ with $p=0.8765, \mathrm{~A}-\mathrm{D}=0.3450$ and Chi-Square distance $=1.9425$ with a corresponding $p=0.7965$. This shows that the Lomax model provides a good fit to the above 
Table 4: Bound length under two-sample approach for parameter $\theta$ when scale parameter $\sigma$ unknown

\begin{tabular}{|c|c|c|c|c|c|c|c|c|c|}
\hline \multicolumn{4}{|c|}{$\theta=2.00$} & \multicolumn{3}{|c|}{ First Future Observation } & \multicolumn{3}{|c|}{ Last Future Observation } \\
\hline$m$ & $t$ & $\sigma$ & $\alpha \downarrow \epsilon \rightarrow$ & $99 \%$ & $95 \%$ & $90 \%$ & $99 \%$ & $95 \%$ & $90 \%$ \\
\hline \multirow{12}{*}{10} & \multirow{6}{*}{04} & \multirow{3}{*}{0.25} & 0.50 & 1.0139 & 0.9824 & 0.9296 & 1.6313 & 1.5809 & 1.5503 \\
\hline & & & 1.10 & 1.1154 & 1.0750 & 1.0253 & 1.7886 & 1.7541 & 1.7057 \\
\hline & & & 1.50 & 1.0123 & 0.9779 & 0.8228 & 1.6262 & 1.5842 & 1.3673 \\
\hline & & \multirow{3}{*}{0.50} & 0.50 & 0.9157 & 0.8972 & 0.8441 & 1.4721 & 1.4559 & 1.4052 \\
\hline & & & 1.10 & 0.9939 & 0.9719 & 0.9205 & 1.5997 & 1.5778 & 1.5338 \\
\hline & & & 1.50 & 0.9082 & 0.8739 & 0.8081 & 1.4585 & 1.4153 & 1.3428 \\
\hline & \multirow{6}{*}{07} & \multirow{3}{*}{0.25} & 0.50 & 0.9919 & 0.9799 & 0.9152 & 1.5966 & 1.5905 & 1.5237 \\
\hline & & & 1.10 & 1.0914 & 1.0705 & 1.0088 & 1.7567 & 1.7380 & 1.6803 \\
\hline & & & 1.50 & 0.9905 & 0.9571 & 0.8055 & 1.5914 & 1.5506 & 1.3387 \\
\hline & & \multirow{3}{*}{0.50} & 0.50 & 0.8955 & 0.8781 & 0.8261 & 1.4409 & 1.4253 & 1.3755 \\
\hline & & & 1.10 & 0.9728 & 0.9511 & 0.9009 & 1.5667 & 1.5444 & 1.5008 \\
\hline & & & 1.50 & 0.8893 & 0.8553 & 0.8222 & 1.4279 & 1.3857 & 1.3666 \\
\hline \multirow{12}{*}{15} & \multirow{6}{*}{04} & \multirow{3}{*}{0.25} & 0.50 & 0.9688 & 0.9569 & 0.8936 & 1.5584 & 1.5528 & 1.4877 \\
\hline & & & 1.10 & 1.0661 & 1.0457 & 0.9854 & 1.7159 & 1.6975 & 1.6411 \\
\hline & & & 1.50 & 0.9671 & 0.9342 & 0.7863 & 1.5534 & 1.5134 & 1.3061 \\
\hline & & \multirow{3}{*}{0.50} & 0.50 & 0.8743 & 0.8573 & 0.8064 & 1.4064 & 1.3913 & 1.3427 \\
\hline & & & 1.10 & 0.9502 & 0.9289 & 0.8796 & 1.5292 & 1.5079 & 1.4651 \\
\hline & & & 1.50 & 0.8678 & 0.8347 & 0.8012 & 1.3934 & 1.3521 & 1.3309 \\
\hline & \multirow{6}{*}{07} & \multirow{3}{*}{0.25} & 0.50 & 0.9479 & 0.9365 & 0.8745 & 1.5252 & 1.5201 & 1.4562 \\
\hline & & & 1.10 & 1.0432 & 1.0231 & 0.9642 & 1.6791 & 1.6613 & 1.6061 \\
\hline & & & 1.50 & 0.9464 & 0.9144 & 0.7697 & 1.5206 & 1.4816 & 1.2791 \\
\hline & & \multirow{3}{*}{0.50} & 0.50 & 0.8556 & 0.8391 & 0.7895 & 1.3768 & 1.3619 & 1.3146 \\
\hline & & & 1.10 & 0.9297 & 0.9091 & 0.8611 & 1.4967 & 1.4764 & 1.4345 \\
\hline & & & 1.50 & 0.8494 & 0.8173 & 0.7761 & 1.3643 & 1.3239 & 1.2898 \\
\hline
\end{tabular}

data. Based on the data selected, all the numerical findings have been calculated for all the considered parametric values and presented in the Tables (8) - (9) for few selected parametric values. All the properties have seen similar as discussed above for all the considered estimation criteria under the simulation. One remarkable point is that, the bound length magnitude has noted wider when they compared to the simulated data for all the considered fixed parametric values. 
Table 5: Bound length under two-sample approach for parameter $\sigma$ when $\sigma$ unknown

\begin{tabular}{|c|c|c|c|c|c|c|c|c|c|}
\hline \multicolumn{4}{|c|}{$\theta=2.00$} & \multicolumn{3}{|c|}{ First Future Observation } & \multicolumn{3}{|c|}{ Last Future Observation } \\
\hline$m$ & $t$ & $\sigma$ & $\alpha \downarrow \epsilon \rightarrow$ & $99 \%$ & $95 \%$ & $90 \%$ & $99 \%$ & $95 \%$ & $90 \%$ \\
\hline \multirow{12}{*}{10} & \multirow{6}{*}{04} & \multirow{3}{*}{0.25} & 0.50 & 0.9523 & 0.9406 & 0.8783 & 1.3267 & 1.3218 & 1.2664 \\
\hline & & & 1.10 & 1.0491 & 1.0292 & 0.9698 & 1.4591 & 1.4436 & 1.3953 \\
\hline & & & 1.50 & 0.9504 & 0.9179 & 0.7717 & 1.3234 & 1.2894 & 1.1134 \\
\hline & & \multirow{3}{*}{0.50} & 0.50 & 0.8588 & 0.8423 & 0.7923 & 1.1976 & 1.1845 & 1.1432 \\
\hline & & & 1.10 & 0.9345 & 0.9138 & 0.8657 & 1.3003 & 1.2825 & 1.2461 \\
\hline & & & 1.50 & 0.8522 & 0.8198 & 0.7578 & 1.1873 & 1.1523 & 1.0934 \\
\hline & \multirow{6}{*}{07} & \multirow{3}{*}{0.25} & 0.50 & 0.9324 & 0.9212 & 0.8603 & 1.2976 & 1.2929 & 1.2387 \\
\hline & & & 1.10 & 1.0275 & 1.0079 & 0.9498 & 1.4271 & 1.4119 & 1.3649 \\
\hline & & & 1.50 & 0.9309 & 0.8993 & 0.7563 & 1.2944 & 1.2615 & 1.0894 \\
\hline & & \multirow{3}{*}{0.50} & 0.50 & 0.8415 & 0.8253 & 0.7764 & 1.1716 & 1.1589 & 1.1184 \\
\hline & & & 1.10 & 0.9156 & 0.8954 & 0.8481 & 1.2723 & 1.2547 & 1.2191 \\
\hline & & & 1.50 & 0.8352 & 0.8033 & 0.7721 & 1.1617 & 1.1274 & 1.1122 \\
\hline \multirow{12}{*}{15} & \multirow{6}{*}{04} & \multirow{3}{*}{0.25} & 0.50 & 0.9096 & 0.8985 & 0.8397 & 1.2677 & 1.2631 & 1.2101 \\
\hline & & & 1.10 & 1.0026 & 0.9835 & 0.9267 & 1.3946 & 1.3796 & 1.3338 \\
\hline & & & 1.50 & 0.9078 & 0.8767 & 0.7378 & 1.2643 & 1.2319 & 1.0636 \\
\hline & & \multirow{3}{*}{0.50} & 0.50 & 0.8205 & 0.8047 & 0.7569 & 1.1443 & 1.1319 & 1.0924 \\
\hline & & & 1.10 & 0.8931 & 0.8733 & 0.8279 & 1.2431 & 1.2258 & 1.1909 \\
\hline & & & 1.50 & 0.8141 & 0.7829 & 0.7511 & 1.1344 & 1.1008 & 1.0838 \\
\hline & \multirow{6}{*}{07} & \multirow{3}{*}{0.25} & 0.50 & 0.8909 & 0.8804 & 0.8221 & 1.2401 & 1.2358 & 1.1838 \\
\hline & & & 1.10 & 0.9818 & 0.9632 & 0.9078 & 1.3643 & 1.3495 & 1.3047 \\
\hline & & & 1.50 & 0.8893 & 0.8599 & 0.7226 & 1.2369 & 1.2053 & 1.0419 \\
\hline & & \multirow{3}{*}{0.50} & 0.50 & 0.8039 & 0.7884 & 0.7418 & 1.1196 & 1.1074 & 1.0693 \\
\hline & & & 1.10 & 0.8758 & 0.8557 & 0.8105 & 1.2168 & 1.1994 & 1.1653 \\
\hline & & & 1.50 & 0.7979 & 0.7674 & 0.7286 & 1.1101 & 1.0773 & 1.0497 \\
\hline
\end{tabular}

Table 6: Asymptotic confidence length for parameter $\theta \& \sigma$

\begin{tabular}{c|c|c|c|c|c|c|c|c}
\hline \multicolumn{3}{c|}{$\theta=2.00$} & \multicolumn{3}{c|}{$\theta$} & \multicolumn{3}{c}{$\sigma$} \\
\hline$\sigma$ & $t$ & $m \downarrow \epsilon \rightarrow$ & $99 \%$ & $95 \%$ & $90 \%$ & $99 \%$ & $95 \%$ & $90 \%$ \\
\hline \multirow{4}{*}{0.25} & \multirow{2}{*}{04} & 10 & 0.9549 & 0.9243 & 0.9131 & 0.9274 & 0.8947 & 0.8834 \\
\cline { 2 - 9 } & \multirow{2}{*}{07} & 15 & 0.8736 & 0.8453 & 0.8203 & 0.8479 & 0.8174 & 0.7802 \\
\cline { 2 - 9 } & & 15 & 0.9331 & 0.9024 & 0.9018 & 0.9061 & 0.8732 & 0.8724 \\
\hline \multirow{3}{*}{0.50} & \multirow{2}{*}{04} & 10 & 0.9528 & 0.8347 & 0.8312 & 0.8275 & 0.7974 & 0.7929 \\
\cline { 2 - 9 } & \multirow{2}{*}{07} & 15 & 0.8331 & 0.8843 & 0.8716 & 0.8859 & 0.8555 & 0.8428 \\
\cline { 2 - 8 } & \multirow{2}{*}{07} & 15 & 0.8912 & 0.8614 & 0.8789 & 0.8082 & 0.7732 & 0.7335 \\
\cline { 2 - 8 } & & 15 & 0.8128 & 0.7903 & 0.7804 & 0.7884 & 0.7543 & 0.7452 \\
\hline
\end{tabular}

Table 7: Repair times of airborne communication transceiver

\begin{tabular}{c|c|c|c|c|c|c}
\hline 0.20 & 0.30 & 0.50 & 0.50 & 0.50 & 0.50 & 0.60 \\
\hline 0.60 & 0.70 & 0.70 & 0.70 & 0.80 & 0.80 & 1.00 \\
\hline 1.00 & 1.00 & 1.00 & 1.00 & 1.10 & 1.30 & 1.50 \\
\hline 1.50 & 1.50 & 1.50 & 2.00 & 2.00 & 2.20 & 2.50 \\
\hline 2.70 & 3.00 & 3.00 & 3.30 & 3.30 & 4.00 & 4.00 \\
\hline 4.50 & 4.70 & 5.00 & 5.40 & 7.00 & 7.50 & 8.80 \\
\hline 9.00 & 10.3 & 22.0 & 24.5 & & & \\
\hline
\end{tabular}


Table 8: Bound lengths under both scenario for real data set with known $\sigma$

\begin{tabular}{|c|c|c|c|c|c|c|c|c|}
\hline \multicolumn{3}{|c|}{$\theta=2.00 \alpha=1.10$} & \multicolumn{3}{|c|}{$\epsilon \downarrow$} & & & \\
\hline$m$ & $t$ & $\sigma$ & $99 \%$ & $95 \%$ & $90 \%$ & $99 \%$ & $95 \%$ & $90 \%$ \\
\hline \multirow{4}{*}{10} & \multirow{2}{*}{04} & 0.25 & 1.3039 & 1.2914 & 1.2244 & & & \\
\hline & & 0.50 & 1.1773 & 1.1463 & 1.1127 & & & \\
\hline & \multirow{2}{*}{07} & 0.25 & 1.2734 & 1.2508 & 1.2151 & & & \\
\hline & & 0.50 & 1.1499 & 1.1192 & 1.0861 & & & \\
\hline \multirow{4}{*}{15} & 04 & 0.25 & 1.2462 & 1.2237 & 1.1989 & & & \\
\hline & $0 t$ & 0.50 & 1.1252 & 1.1048 & 1.0725 & & & \\
\hline & 07 & 0.25 & 1.2171 & 1.1847 & 1.1607 & & & \\
\hline & 01 & 0.50 & 1.0989 & 1.0687 & 1.0472 & & & \\
\hline & & & \multicolumn{3}{|c|}{ First Future Observation } & \multicolumn{3}{|c|}{ Last Future Observation } \\
\hline \multirow{4}{*}{10} & \multirow{2}{*}{04} & 0.25 & 1.1512 & 1.1294 & 1.0642 & 1.6025 & 1.5756 & 1.5326 \\
\hline & & 0.50 & 1.0251 & 1.0025 & 0.9497 & 1.4279 & 1.4084 & 1.3685 \\
\hline & \multirow{2}{*}{07} & 0.25 & 1.1274 & 1.0968 & 1.0422 & 1.5673 & 1.5407 & 1.4991 \\
\hline & & 0.50 & 1.0043 & 0.9722 & 0.9303 & 1.3971 & 1.3678 & 1.3388 \\
\hline \multirow{4}{*}{15} & \multirow{2}{*}{04} & 0.25 & 1.1098 & 1.0691 & 1.0168 & 1.5316 & 1.5052 & 1.4649 \\
\hline & & 0.50 & 0.9796 & 0.9679 & 0.9072 & 1.3654 & 1.3467 & 1.3077 \\
\hline & \multirow{2}{*}{07} & 0.25 & 1.0772 & 1.0468 & 0.9969 & 1.4981 & 1.4721 & 1.4329 \\
\hline & & 0.50 & 0.9602 & 0.9386 & 0.8889 & 1.3354 & 1.3078 & 1.2796 \\
\hline
\end{tabular}

Table 9: Bound lengths under both scenario for real data set with unknown $\sigma$

\begin{tabular}{|c|c|c|c|c|c|c|c|c|}
\hline \multicolumn{3}{|c|}{$\theta=2.00, \alpha=1.10$} & \multicolumn{3}{|c|}{ OS-BPB $(\theta) \epsilon \downarrow$} & \multicolumn{3}{|c|}{ OS-BPB $(\sigma) \epsilon \downarrow$} \\
\hline$m$ & $t$ & $\sigma$ & $99 \%$ & $95 \%$ & $90 \%$ & $99 \%$ & $95 \%$ & $90 \%$ \\
\hline \multirow{4}{*}{10} & \multirow{2}{*}{04} & 0.25 & 1.2804 & 1.2381 & 1.1512 & 1.3345 & 1.3076 & 1.1756 \\
\hline & & 0.50 & 1.1294 & 1.1056 & 1.0809 & 1.1772 & 1.1504 & 1.1241 \\
\hline & \multirow{2}{*}{07} & 0.25 & 2498 & 1.2018 & 1.1836 & 1.3026 & 1.2671 & 1.2291 \\
\hline & & 0.50 & 1137 & 1.0997 & 1.0755 & 1.1606 & 1.1435 & 1.0981 \\
\hline \multirow{4}{*}{15} & \multirow{2}{*}{04} & 0.25 & 2224 & 1.2002 & 1.1786 & 1.2741 & 1.2408 & 1.2036 \\
\hline & & 0.50 & 1.0896 & 1.0647 & 1.0325 & 1.1304 & 1.1078 & 1.0746 \\
\hline & \multirow[b]{2}{*}{07} & 0.25 & 1.1952 & 1.1731 & 1.1512 & 1.2456 & 1.2108 & 1.1756 \\
\hline & & 0.50 & 1.0643 & 1.0451 & 1.0277 & 1.1079 & 1.0735 & 1.0493 \\
\hline \multicolumn{3}{|c|}{ For Parameter $\theta$} & \multicolumn{3}{|c|}{ First Future Observation } & \multicolumn{3}{|c|}{ Last Future Observation } \\
\hline \multirow{4}{*}{10} & \multirow{2}{*}{0} & 0.25 & 1.2301 & 1.1854 & 1.1306 & 1.7841 & 1.7497 & 1.7015 \\
\hline & & 0.50 & 1.0951 & 1.0712 & 1.0142 & 1.5951 & 1.5734 & 1.5296 \\
\hline & \multirow{2}{*}{07} & 0.25 & 2033 & 1.1804 & 1.1123 & 1.7521 & 1.7336 & 1.6761 \\
\hline & & 0.50 & 1.0717 & 1.0479 & 0.9925 & 1.5621 & 1.5401 & 1.4966 \\
\hline \multirow{4}{*}{15} & \multirow{2}{*}{04} & 0.25 & 1.1753 & 1.1529 & 1.0863 & 1.7113 & 1.6931 & 1.6369 \\
\hline & & 0.50 & 0467 & 1.0233 & 0.9688 & 1.5246 & 1.5035 & 1.4609 \\
\hline & \multirow[b]{2}{*}{07} & 0.25 & 1.1498 & 1.1278 & 1.0627 & 1.6745 & 1.6569 & 1.6019 \\
\hline & & 0.50 & 1.0239 & 1.0013 & 0.9483 & 1.4921 & 1.4722 & 1.4303 \\
\hline \multicolumn{3}{|c|}{ For Para } & \multicolumn{3}{|c|}{ First Future Observation } & \multicolumn{3}{|c|}{ Last Future Observation } \\
\hline \multirow{4}{*}{10} & \multirow{2}{*}{04} & 0.25 & 1.1628 & 1.1403 & 1.0846 & 1.8927 & 1.7893 & 1.6862 \\
\hline & & 0.50 & 1.0355 & 1.0119 & 0.9679 & 1.6865 & 1.5894 & 1.5057 \\
\hline & \multirow{2}{*}{07} & 0.25 & 1.1388 & 1.1164 & 1.0622 & 1.8511 & 1.7501 & 1.6494 \\
\hline & & .50 & & & & 1.6501 & 1.5549 & 1.4731 \\
\hline \multirow{4}{*}{15} & \multirow{2}{*}{0} & 0.25 & 112 & 1.0893 & 1.0363 & 1.8089 & 1.7099 & 1.6118 \\
\hline & & 0.50 & 0.9895 & 0.9669 & 0.9256 & 1.6122 & 1.5191 & 1.4389 \\
\hline & \multirow{2}{*}{07} & 0.25 & 1.0881 & 1.0667 & 1.0151 & 1.7696 & 1.6725 & 1.5766 \\
\hline & & 0.50 & 0.9703 & 0.9474 & 0.9061 & 1.5781 & 1.4863 & 1.4079 \\
\hline
\end{tabular}




\section{References}

Ahmad A, Ahmad SP, Ahmed A (2016). "Bayesian Analysis of Lomax Distribution under Asymmetric Loss Functions." Journal of Statistics Applications and Probability Letters, 3(1), 35-44. URL http://dx.doi.org/10.18576/jsapl/030105.

Balakrishnan N, Cramer E (2014). The Art of Progressive Censoring: Applications to Reliability and Quality. BirkhÃd'user, Boston.

Chhikara RS, Folks JL (1977). "The Inverse Gaussian Distribution as a Lifetime Model." Technometrics, 19, 461-468. URL http://dx.doi.org/10.2307/1267886.

Harries CM (1967). "Queues with Stochastic Service Rate." Navel Research Logistics Quarterly, 14, 219-230.

Hastings WK (1970). "Monte Carlo Sampling Methods using Markov Chains and Their Applications." Biometrika, 57, 97-109. URL http://dx.doi.org/10.2307/2334940.

Kayal T, Tripathi YM, Rastogi MK, Asgharzadeh A (2017). "Inference for Burr XII Distribution under Type-I Progressive Hybrid Censoring." Communication in Statistics - Simulation and Computation, 46(9), 7447-7465. URL https://doi.org/10.1080/03610918. 2016.1241405.

Kundu D, Joarder A (2006). "Analysis of Type-II Progressively Hybrid Censored Data." Computational Statistics and Data Analysis, 50, 2509-2528. URL https://doi.org/10. 1016/j.csda.2005.05.002.

Lin CT, Ng HKT, Chan PS (2009). "Statistical Inference of Type-II Progressively Hybrid Censored Data with Weibull Lifetimes." Communications in Statistics - Theory and Methods, 38, 1710-1729. URL https://doi.org/10.1080/03610920902850069.

Lomax KS (1954). "Business Failures. Another Example of the Analysis of Failure Data." Journal of the American Statistical Association, 49, 847-852. URL http://dx.doi.org/ $10.2307 / 2281544$.

Madi MT, Raqab MZ (2004). "Bayesian Prediction of Temperature Records Using the Pareto Model." Environmetrics, 15(7), 701-710. URL https://doi.org/10.1002/env.661.

Metropolis N, Rosenbluth AW, Rosenbluth MN, Teller AH, Teller E (1953). "Equations of State Calculations by Fast Computing Machines." Journal of Chemical Physics, 21, 10871092.

Okasha HM (2014). "E-Bayesian Estimation for the Lomax Distribution Based on Type-II Censored Data." Journal of the Egyptian Mathematical Society, 22(3), 489-495. URL https://doi.org/10.1016/j.joems.2013.12.009.

Park S, Balakrishnan N (2009). "On Simple Calculation of the Fisher Information in Hybrid Censoring Schemes." Statistics and Probability Letters, 79, 1311-1319. URL https://doi. org/10.1016/j.spl.2009.02.004.

Prakash G (2014). "Right Censored Bayes Estimator for Lomax Model." Statistics Research Letters, 3(1), 23-28.

Prakash G (2017). "Some Bayes Estimators for Pareto Type-II Progressive Censored Data." Electronic Journal of Applied Statistical Analysis, 10(1), 257-270. URL https://doi .org/ 10.1285/i20705948v10n1p257.

Singh B, Gupta PK, Sharma VK (2014). "On Type-II Hybrid Censored Lindley Distribution." Statistics Research Letters, 3, 58-62. 
Steindle J (1965). Random Processes and the Growth of Firms, A Study of the Pareto Law. Heffner, New York.

Wei S, Wang C, Li Z (2017). "Bayes Estimation of Lomax Distribution Parameter in the Composite LINEX Loss of Symmetry." Journal of Interdisciplinary Mathematics, 20(5), 1277-1287. URL https://doi.org/10.1080/09720502.2017.1311043.

\section{Affiliation:}

Gyan Prakash

Department of Community Medicine,

M. L. N. Medical College, Allahabad, U. P., India.

E-mail: ggyanji@yahoo.com

\section{Austrian Journal of Statistics}

published by the Austrian Society of Statistics

Volume 49

February 2020 http://www .ajs.or.at/

http://www.osg.or.at/

Submitted: 2018-10-10

Accepted: 2019-01-25 